\title{
Contact-based navigation for an autonomous flying robot
}

\author{
Adrien Briod ${ }^{1}$, Przemyslaw Kornatowski ${ }^{1}$, Adam Klaptocz ${ }^{3}$, Arnaud Garnier ${ }^{2}$, Marco Pagnamenta ${ }^{2}$, \\ Jean-Christophe Zufferey ${ }^{3}$ and Dario Floreano ${ }^{2}$
}

\begin{abstract}
Autonomous navigation in obstacle-dense indoor environments is very challenging for flying robots due to the high risk of collisions, which may lead to mechanical damage of the platform and eventual failure of the mission. While conventional approaches in autonomous navigation favor obstacle avoidance strategies, recent work showed that collisionrobust flying robots could hit obstacles without breaking and even self-recover after a crash to the ground. This approach is particularly interesting for autonomous navigation in complex environments where collisions are unavoidable, or for reducing the sensing and control complexity involved in obstacle avoidance. This paper aims at showing that collision-robust platforms can go a step further and exploit contacts with the environment to achieve useful navigation tasks based on the sense of touch. This approach is typically useful when weight restrictions prevent the use of heavier sensors, or as a low-level detection mechanism supplementing other sensing modalities.

In this paper, a solution based on force and inertial sensors used to detect obstacles all around the robot is presented. Eight miniature force sensors, weighting $0.9 \mathrm{~g}$ each, are integrated in the structure of a collision-robust flying platform without affecting its robustness. A proof-of-concept experiment demonstrates the use of contact sensing for exploring autonomously a room in 3D, showing significant advantages compared to a previous strategy. To our knowledge this is the first fully autonomous flying robot using touch sensors as only exteroceptive sensors.
\end{abstract}

\section{INTRODUCTION}

Flying robots have unique advantages in the exploration and surveillance of indoor environments presenting dangers to humans, such as caves, semi-collapsed buildings or radioactive areas. Flight as indoor locomotion is interesting because it is not constrained by the morphology of the ground and can be used to navigate over obstacles more efficiently than ground-based locomotion. Current flying systems however have difficulty in dealing with the large amount of obstacles inherent to such environments. Collisions with this 'clutter' usually result in crashes from which the platform can no longer recover.

A lot of research is therefore focused on obstacle detection and avoidance, using for example vision [1], IR range sensors [2] or lasers [3], sometimes coupled with powerful algorithms such as SLAM [4], [5], [6]. However, the lack of global positioning (like GPS) and the unstable nature of flying platforms render this task increasingly difficult as the complexity of the environment increases, requiring advanced

This work was supported by the Swiss National Science Foundation through the National Centre of Competence in Research (NCCR) Robotics.

Authors are with: (1) the Laboratory of Intelligent Systems (LIS) http://lis.epfl.ch, Ecole Polytechnique Fédérale de Lausanne (EPFL), 1015 Lausanne, Switzerland, (2) EPFL, (3) SenseFly Ltd, 1024 Ecublens, Switzerland

Contact e-mail: adrien.briod@epfl.ch.

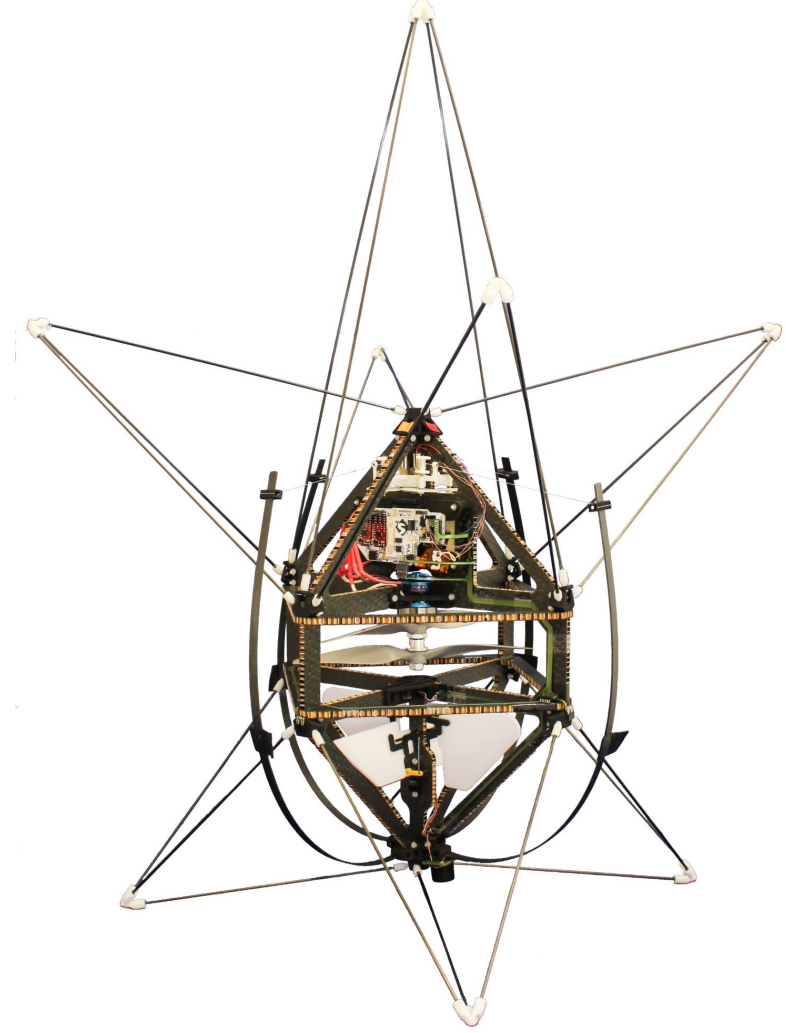

Fig. 1. The AirBurr: a 350g flying robot that can sustain collisions and c an self-recover once on the ground thanks to active legs. Such a platform is not afraid of collision with obstacles, and can thus even exploit contacts with the environment and achieve navigation tasks only based on the sense of touch, as is demonstrated in this paper.

sensors, powerful processors and precise mapping of the environment. Due to their weight, such platforms are usually fragile and prone to mission-ending crashes if a collision happens with an obstacle that failed to be detected by the sensors.

A new approach aiming at improving the robustness and locomotion efficiency of flying platforms in cluttered environments was introduced recently in [7]. This approach takes inspiration from insects which, despite their high agility, are not able to avoid all obstacles but can typically recover quickly in the air and continue flying after collisions [8]. Similarly, it is suggested to use platforms that can sustain collisions and can remain stable in the air after impacts or self-recover once on the ground. Because collisions are acceptable, such platforms can locomote efficiently through cluttered environments without the caution and low speed 
often required for sense and avoid approaches. Obstacles do not need to be perfectly (or even at all) detected, which reduces drastically the complexity and weight of the embedded sensors required for navigation. Finally, such robots are more robust to moving obstacles, sensor failures or unexpected situations.

Results obtained in [9] show how such a platform (pictured in figure 1) can fly with minimal sensing in unknown environments by going towards its goal until it collides with an obstacle, falling to the ground without breaking, recovering thanks to active legs and taking-off again to continue its mission. More information about the collision absorption and self-recovery mechanisms can be found in [10] and [11] respectively. Basic contact sensing was implemented in the past thanks to accelerometers, which can only detect high impact forces and had thus several drawbacks:

- The robot could get stuck against an obstacle without detecting it, either because the obstacle was soft, or because the robot collided at low speed.

- When the speed of the robot was set high enough to always provoke detectable collisions, the chances for the robot to stabilize in flight after a collision diminished. In fact, the strategy chosen in [9] was to turn off the motor after each collision because collisions were usually too strong to subsequently stabilize the robot in flight .

While this navigation strategy allowed the robot to explore autonomously a room or cross a corridor, it was not time efficient due to the numerous falls to the ground and subsequent self-recovery procedures, or because the robot would sometimes stay against an obstacle for long periods of time without detecting it.

In this paper, we suggest to augment the platform pictured in fig 1 with the sense of touch, and exploit collisions similarly to a flying insect that repeatedly collides with a window until it finds an escape route. Instead of falling to the ground when a collision is detected, we suggest that we can stay in the air most of the time and learn from the information collected during the collision to control the robot. Touch is commonly used on ground robots as sole sensors for 2D navigation [12] or as a complementary modality to additional positioning sensors [13]. Navigation strategies using only touch sensing include area coverage tasks, for example for vacuum cleaning [14], or obstacle following [15]. Some systems equipped with distance or positioning sensors use the sense of touch to detect unpredictable or moving obstacles that may not be detected by the other sensors [13], [16]. We suggest that several strategies that have been extensively researched for ground robots can be extended to flying robots navigating in 3D and that touch sensing will allow flying robots to perform certain navigation tasks or deal with hardto-detect obstacles.

As a proof-of-concept demonstration, we show that a contact-sensitive flying robot is able to explore fully autonomously a closed environment in 3D solely using the sense of touch. In the process, we show that thrust regulation, which is a critical problem for autonomous flying robots and usually addressed with a range sensor, can be solved when generalizing contact-based navigation to $3 \mathrm{D}$.

\section{CONTACT SENSING}

Sensors have to be used to know if a platform is in contact with an obstacle, and where the obstacle is. There are several ways to obtain that information, typically found on ground robots, such as tactile sensors at the interface [17], whiskers [18] or proximity sensors [19]. It is also possible to detect contacts with obstacle by comparing the expected motion of a platform and the actual motion, like in [15] where collisions are detected when the odometry measurements do not match the expectation. This can be referred to as the proprioceptive sense.

Several problems are exacerbated when designing contact sensing solutions for flying platforms:

- The sensors must be lightweight, since payload is limited on flying robots.

- Because a flying robot is moving in 3 dimensions, the sensors have to detect collisions that may happen on all sides of a volume, as opposed to the perimeter for ground robots.

- The sensors should not affect the robustness of the protective structure, which is generally very stiff in order to absorb collision energy [10].

- The sensors have to sustain the strong forces occurring when the flying platform collides with obstacles at high speed or falls to the ground.

- The sensors need a high sensitivity to detect light contacts. Typically, in case of static contact with an obstacle, the interaction force $\mathbf{F}_{\mathbf{s}}$ is equivalent to the horizontal component of the lift force, which is computed as

$$
\mathbf{F}_{\mathbf{s}}=m \cdot g \cdot \tan \alpha
$$

where $m$ is the robot's mass, $g$ is the earth's gravity constant and $\alpha$ the lean angle of the robot against the wall. Since the lean angle may be just a few degrees,

$\mathbf{F}_{\mathbf{s}}$ can be reduced to a small fraction of the lift force.

While the accelerometers used in past experiments fulfill several of the challenges presented above, we show in the following subsection that they are too limited in terms of sensitivity and that additional sensors are required.

\section{A. Accelerometers}

Accelerometers are standard motion sensors found on most flying robots and thus don't involve any extra hardware, which makes them a logical initial solution for contact sensing. It is thus interesting to study their capabilities and limitations. Accelerometers can detect contacts thanks to the proprioceptive sense. When the gravity component is removed from the raw measurements $\mathbf{a}_{\text {raw }}$, they give the 3 axis linear accelerations $a_{\text {lin }}$ corresponding to the robot's own motion. $a_{\text {lin }}$ can be obtained using the following formula:

$$
\mathbf{a}_{\text {lin }}=\mathbf{a}_{\text {raw }}-\mathbf{R}^{-\mathbf{1}} \cdot\left[\begin{array}{c}
0 \\
0 \\
-g
\end{array}\right]
$$




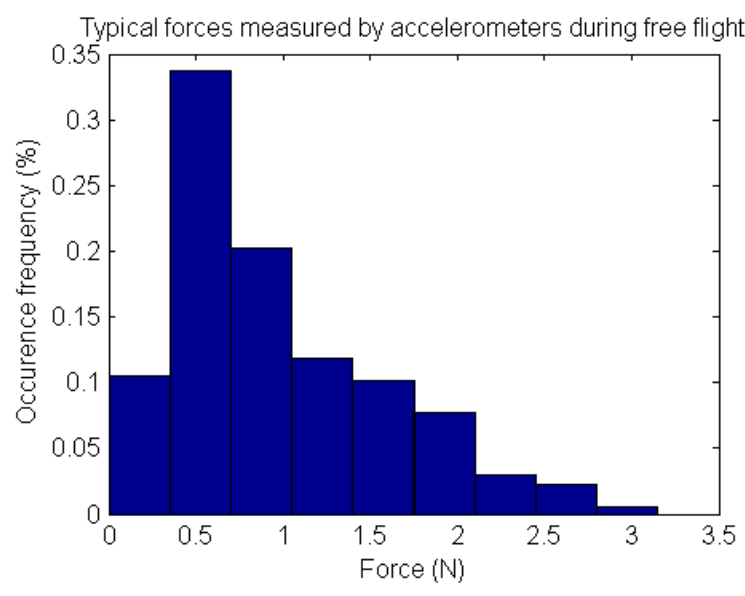

Fig. 2. Histogram showing the distribution of the forces affecting the flying platform during free flight, obtained from the measured linear accelerations and equations (2)-(3). These forces result from the robot's own actuators, from aerodynamic forces or gravitational pull. It can be seen that during normal free flights, the force never goes above $3.5 \mathrm{~N}$. It is assumed that forces larger than $3.5 \mathrm{~N}$ are thus generated by collisions with external obstacles.

where $\mathbf{R}$ is the rotation matrix describing the orientation of the robot and $g$ is the earth's gravity constant. The orientation of the robot is typically obtained by sensor fusion of accelerometers, gyroscopes and magnetometers in a way that perturbations from collisions do not disturb the orientation estimation but this is out of the scope of this publication. The resultant $\mathbf{F}_{\text {ext }}$ of all external forces affecting the platform's motion can be retrieved according to the following formula (Newton's laws of motion):

$$
\mathbf{F}_{\text {ext }}=m \cdot \mathbf{a}_{\text {lin }}
$$

Contacts can be detected by calculating the difference between the expected force and the actual measured force affecting the platform's motion. The difference corresponds to an additional external force, assumed to be generated by a contact with an obstacle. Calculating expected forces during flight requires a good model of the platform's actuators and aerodynamic forces, which can be hard to obtain. As a simple solution, we suggest to detect when the measured force rises above the values typically found during free-flight and label that as a collision. In order to define an appropriate threshold, typical forces generated during free-flight were analyzed (see figure 2). A threshold of $3.5 \mathrm{~N}$ was chosen for our specific platform.

To estimate where around the robot the obstacle is, it is assumed that it is directly aligned with the force vector. This assumption would only be true if the robot's structure was a frictionless sphere, but it is the best approximation possible. A characterization of obstacle position detection is presented in subsection II-C.

The accelerometer-based approach for contact detection can only be used when collisions with obstacles generate forces above $3.5 \mathrm{~N}$, which is often true during dynamic contacts occurring when the platform collides at significant speed. However, when colliding at low speed or on soft obstacles, the forces may be much smaller. In particular, during static contacts where the contact force is only created by the lift force, as described by equation (1), forces as small as $0.15 \mathrm{~N}$ have to be detected (value obtained for lean angle $\alpha=3.5^{\circ}$ and $m=350 \mathrm{~g}$. This is why more sensitive sensors are required for these situations.

\section{B. Force sensors}

In order to detect static contacts with obstacles, sensitive force sensors were designed and integrated to the existing protective structure of the AirBurr. The structure was designed to absorb collision energy thanks to 24 buckling springs arranged in 8 tetrahedral configurations [10], as can be seen on figure 3. One force sensor was integrated in each of these tetrahedra - or bumpers - to make them contact sensitive, since they are the parts likely to touch obstacles. The 8 pivot joints indicated in figure 3 have been replaced by the sensors pictured in figure 4 . These sensors measure the deformation of low stiffness springs thanks to hall sensors and solve all the challenges described at the beginning of the section: they allow to obtain a high sensitivity while conserving the stiff buckling spring mechanism for collision energy absorption. Also, they survived numerous crashes without breaking, and each sensor weighs only $0.9 \mathrm{~g}$.

The spring stiffness, magnet and travel distance were dimensioned thanks to a model of the magnetic field so as to maximize the sensitivity of the force measurement and provide a range of $\pm 1 N$. Figure 5 shows the output signal of the sensor with respect to a longitudinal force. Some hysteresis was observed in practice, due to the friction between the two moving parts, which prevents them to come back to the exact same original position after a contact. This problem is corrected in software by resetting the zero offset after each contact. A picture of the sensor mounted on the real robot is shown in figure 6. Tests in flight allowed to measure the standard deviation of the sensor in a real situation, in order to estimate the sensitivity of the force sensing. It was found that the standard deviation of the measures when no force is applied is generally around $\sigma=0.01 N$. A threshold of $3 \sigma$ was used to detect contacts, which means the sensors are sensitive to axial forces as small as $0.03 \mathrm{~N}$.

Using only one sensor for each bumper does not allow to measure exactly the contact forces, except if the force is exactly aligned with the carbon rod. However, it allows to detect whether the bumper is in contact with an obstacle or not, it gives an approximation of the contact force, and the sign of the deformation allows to detect if the contact comes from the side, top (for the above sensors) or bottom (for the lower sensors). The configuration of the bumpers allow to distinguish obstacles between 4 different sides. It should be noted that the same sensor could potentially equip all 24 buckling springs ( 3 sensors per bumper), which would allow to know the complete 3D force information applied on each bumper. 


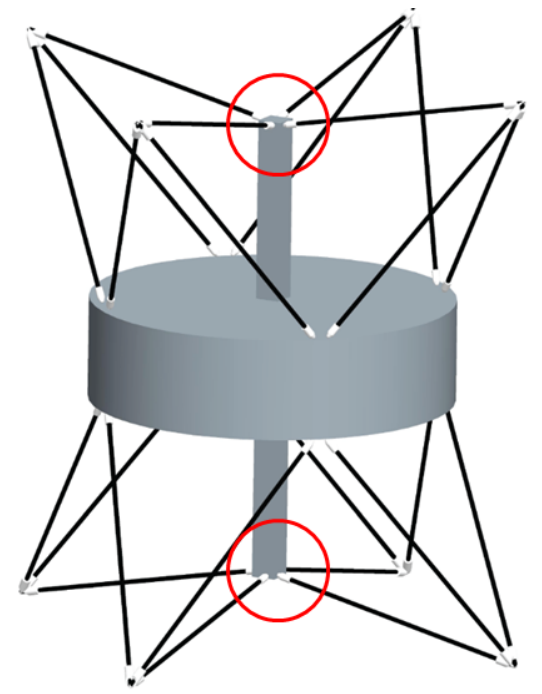

Fig. 3. Simplified picture of the protective structure of the collision robust flying platform used in this paper, based on 24 buckling springs arranged in 8 tetrahedra (or bumpers). Each buckling spring is made from a pulltruded carbon fiber rod and is connected at its ends with a passive pivot joint (more information can be found in [10]). The circles indicate the 8 joints where force sensors are added.

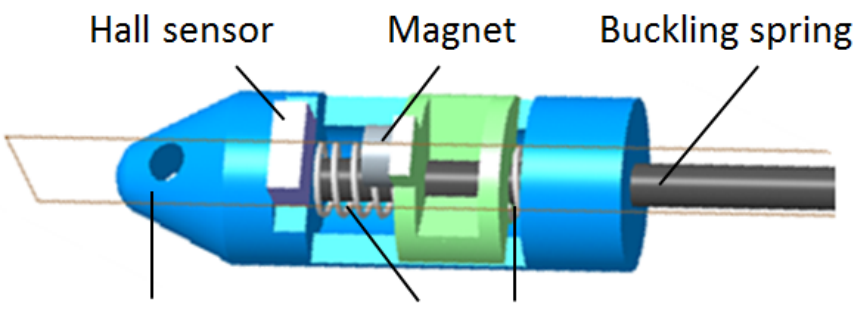

Pivot joint

Helical springs
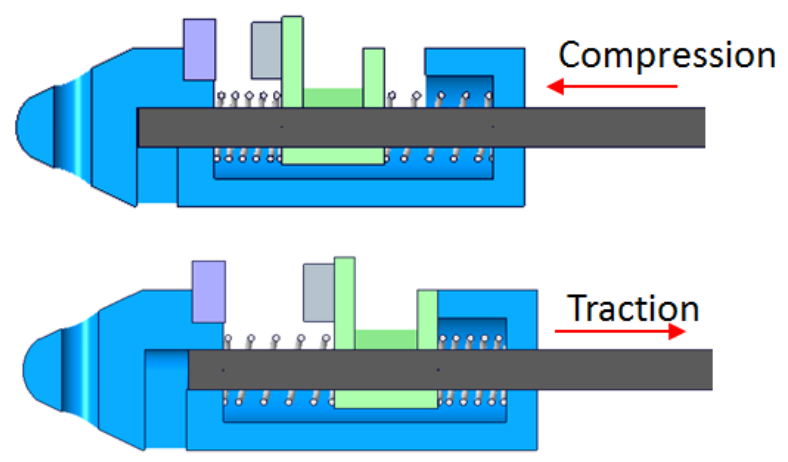

Fig. 4. Force sensors integrated in the pivot joint of the buckling springs shown in figure 3. Two low stiffness helical springs are mounted in series to the carbon fiber buckling spring, so that they deform first when an obstacle touches the bumper. The low stiffness springs' range of motion is $1 \mathrm{~mm}$ in both directions, after which the buckling spring is mechanically stopped and takes the force. The deformation of the low stiffness springs is measured thanks to a fixed hall sensor and a magnet on the moving part. The additional weight compared to the original pivot joint is $0.9 \mathrm{~g}$. When used in the structure shown in figure 3, these sensors allow to differentiate contacts from the side or from the top (for above sensors) or bottom (for lower sensors) because side contacts exert compression on the rods, and traction in the other cases.

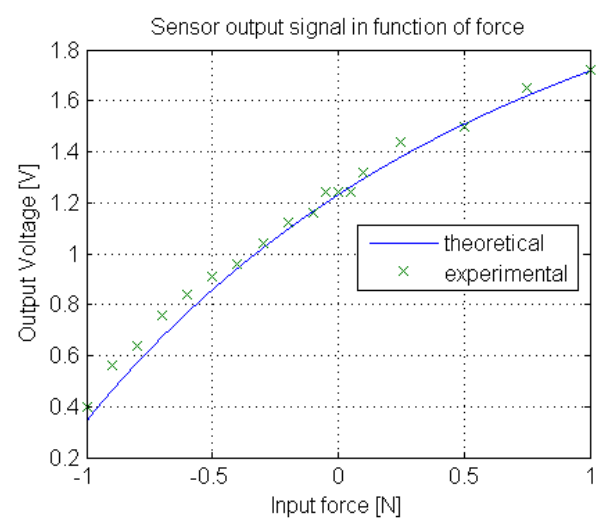

Fig. 5. Curve showing the relation between the hall sensor output and the force applied on the bumper, based on a model of the magnetic field. An experimental validation of this model was carried out using an external force sensor as a ground truth.

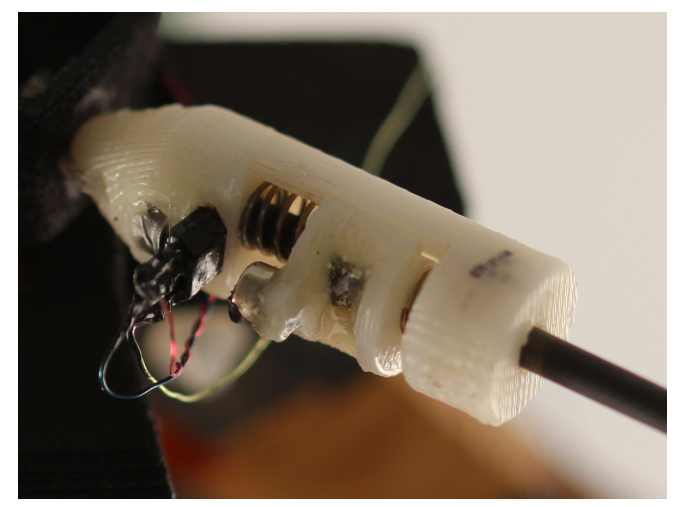

Fig. 6. Photo of the force sensor described in fig 4, as mounted on the flying robot. The parts were produced thanks to a $3 \mathrm{D}$ printer.

\section{Characterization of obstacle position measurement}

In order to assess the capability of the accelerometers and force sensors to detect where obstacles are around the robot, a simple experiment is performed. The flying platform is remotely controlled to perform multiple side collisions on a wall of known orientation. The robot's orientation is known thanks to the onboard IMU, which allows to calculate the ground truth of where the obstacle is with respect to the platform's local frame. About 125 collisions of various intensities were detected by the bumpers, and the position of the obstacle was estimated every time on the robot using both the accelerometers and the bumpers. The errors are plotted in figure 7 versus the contact intensity, which shows that the bumpers have a constant accuracy regardless of the contact force, but the accelerometers only give comparable results for contact intensities higher than $4 N$. This can be explained because accelerometer-based detection is affected by additional forces that are not related to the collision as discussed in subsection II-A. Therefore the accuracy is only good from a certain intensity where the contact force is significantly larger than the others. These results show that the bumpers can always be trusted to give the position of an obstacle around the robot, whereas the accelerometer can 


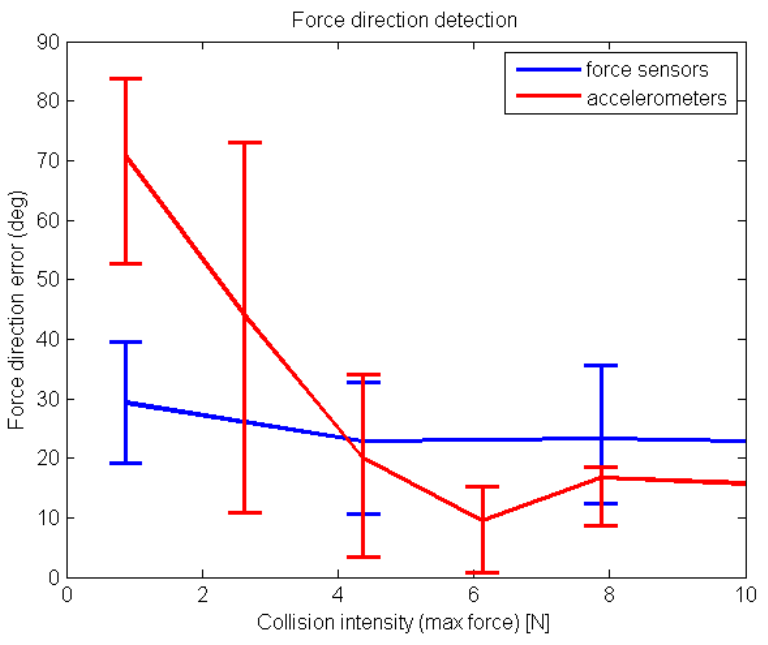

Fig. 7. Characterization of the obstacle position detection by the accelerometers and the bumpers, in function of the contact intensity. The obstacle position is expressed as a direction pointing to the obstacle from the robot's center. The graph obtained from 125 collisions shows that the accelerometers start to be more reliable than the bumpers for collision intensities higher than $4 \mathrm{~N}$.

give a better estimate if the contact intensity is higher than a threshold. This information is used later in the controller, to know which sensor to trust in function of the contact intensity.

\section{AUTONOMOUS NAVIGATION}

We show here that contact sensors as only exteroceptive sensors can be used for autonomous navigation. A 3-dimension exploration strategy is demonstrated in a $3.5 \times 6 \times 2.7 \mathrm{~m}$ room and improves significantly on previous results [9]. Also the use of contact sensors for thrust control is demonstrated.

The collision robust flying robot shown in figure 1, augmented with the sensors presented in section II-B is used for the experiments. The uprighting mechanism (legs) and top protection part shown in the picture are not used in these experiments for practical reasons, and the robot is manually uprighted in the rare event that a fall to the ground occurs.

The robot is equipped with two contra-rotating propellers for thrust and yaw control, while two control surfaces placed under the rotors are used for orientation stabilization. The robot is capable of stable vertical hovering thanks to an embedded IMU and an attitude controller. In order to improve the chances of staying in the air after a collision with an obstacle, the attitude stabilization controller's response time was optimized.

The navigation algorithm uses a set of open-loop behaviors to control the robot towards a desired direction in 3D. To move sideways towards a desired direction $\Psi_{d}$, commands in roll $\phi_{d}$ and pitch $\theta_{d}$ are given to the attitude controller according to the following formulas:

$$
\begin{aligned}
\phi_{d} & =\alpha \cdot \sin \Psi_{d} \\
\theta_{d} & =-\alpha \cdot \cos \Psi_{d}
\end{aligned}
$$

where $\alpha$ is the lean angle of the robot, which is used to tune the speed of the sideways motion. To move up or down, two different thrust commands that are a little higher or lower than the hovering command are sent to the motors. The hovering command, that keeps approximately the robot at a constant altitude, has to be calibrated in advance. While these open-loop behaviors allow to control approximately the flight direction of the robot in 3D, they are very imprecise compared to closed-loop solutions using speed or position sensors.

As a proof of concept, a very simple navigation strategy was programmed for autonomous random exploration. Like for ground robots achieving similar tasks [14], the principle is that once an obstacle is detected, the robot picks a direction pointing away from the obstacle. After each sideways collision, the navigation algorithm updates the desired flight direction $\Psi_{d}$ so that it points away from the estimated obstacle position. After each collision detected at the top or bottom of the robot, the navigation algorithm picks the thrust command to go down or up respectively.

In order to visualize the $3 \mathrm{D}$ trajectory, a tracking system composed of two wide-angle cameras mounted in the ceiling were used. The tracking of the robot on each image is achieved by background subtraction, and the 3D position is calculated by triangulation. While the precision varies from several centimeters in the best case to a few tens of centimeters in the worst case, the 3D trajectory is still useful for visualization purposes.

The trajectory of the robot performing random navigation during 260 seconds is shown in figure 8. During the flight, it recovered in the air from more than 120 collisions, which were detected by the sensors and were subsequently used by the navigation algorithm to determine new direction commands. The robot failed to recover in the air from 3 collisions which led to falls to the ground and manual uprighting.

These results are significantly better than the previous exploration strategy from [9] for the following reasons:

- Most collisions do not lead to a crash to the ground, unlike previously, which means that less time is spent for self-recovery and the time spent exploring the room rises to $80 \%$ (against $25 \%$ previously). This means that the new strategy exploiting contacts allows to cover approximately 3 times more distance in average per unit of time. Additionally, less falls to the ground reduce the risks of breaking the platform, or getting stuck while self-recovering.

- The contact-sensitive robot is much less likely to get stuck against an obstacle while flying than the previous platform which would fail to detect some obstacles.

- The height was previously controlled thanks to an ultrasonic sensor, which is not required anymore. Also, the exploration is now covering the whole $3 \mathrm{D}$ space, whereas it was constrained to a plane at constant height previously. 


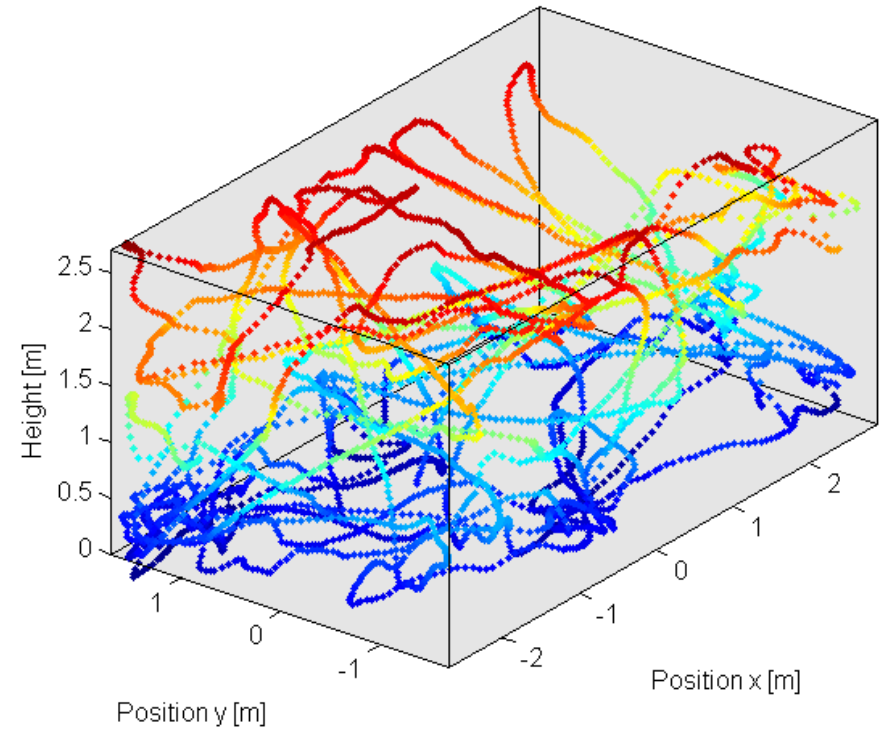

Fig. 8. 3D plot of the trajectory of the robot performing random exploration of a $3.5 \times 6 \times 2.7 \mathrm{~m}$ room. The robot uses the information from contact sensors to correct its direction after every collision with obstacles, be it with walls, the ceiling or the floor. The trajectory is plotted with different colors depending on the height. During the total flight time of 260 seconds, the robot reacted to more than 120 contacts with obstacles. This is to our knowledge the first time a flying robots navigates fully autonomously using solely the sense of touch.

\section{CONCLUSION}

The whole structure of a collision robust flying robot was made contact sensitive thanks to 8 miniature force sensors weighing $0.9 \mathrm{~g}$ each. A navigation strategy allows to react to collisions with obstacles and explore randomly a room in 3 dimensions. The new contact-based exploration is a significant improvement over the previous results obtained without touch sensors in [9]. This proof-of-concept experiment shows that it is possible to control a flying robot solely using the information from contacts with obstacles. This first step opens the door to new simple yet robust navigation strategies that exploit the interactions with the environment and that were only used by ground robots up to now. While many applications may require the use of more advanced sensors for different navigation tasks, the results presented in this paper show that contact sensors have their use on flying robots and could be considered in the future as part of a multi-modal sensing strategy.

Future work will concentrate on improving the stabilization of the robot after it collides with obstacles, so that it can use this strategy in cluttered environments without risking to fall to the ground. In order to tackle more advanced behaviors, sensors for speed estimation will be investigated to enable closed-loop motion control and short term position estimation. This will allow to map detected obstacles in the short term, determine the approximate size of the environment, and perform precise maneuvers such as following a wall or finding a way through clutter.

\section{REFERENCES}

[1] J.-C. Zufferey, A. Beyeler, and D. Floreano, "Optic Flow to Steer and Avoid Collisions in 3D," Flying Insects and Robots, pp. 73-86, 2009.

[2] J. F. Roberts, T. S. Stirling, J.-c. Zufferey, and D. Floreano, "Quadrotor Using Minimal Sensing For Autonomous Indoor Flight," in European Micro Air Vehicle Conference and Flight Competition (EMAV2007), no. September, 2007, pp. 17-21.

[3] D. Schafroth, S. Bouabdallah, C. Bermes, and R. Siegwart, "From the Test Benches to the First Prototype of the muFly Micro Helicopter," Journal of Intelligent and Robotic Systems, vol. 54, no. 1-3, pp. 245-260, Jul. 2008.

[4] R. He, A. Bachrach, and N. Roy, "Efficient Planning under Uncertainty for a Target-Tracking Micro-Aerial Vehicle," Distribution, pp. 1-8, 2010.

[5] S. Shen, N. Michael, and V. Kumar, "Autonomous multi-floor indoor navigation with a computationally constrained MAV," in Robotics and Automation (ICRA), 2011 IEEE International Conference on. IEEE, 2011, pp. 20-25.

[6] D. Scaramuzza, M. C. Achtelik, L. Doitsidis, F. Fraundorfer, E. B. Kosmatopoulos, A. Martinelli, M. W. Achtelik, M. Chli, S. A. Chatzichristofis, L. Kneip, D. Gurdan, L. Heng, G. H. Lee, S. Lynen, L. Meier, M. Pollefeys, R. Siegwart, J. C. Stumpf, P. Tanskanen, C. Troiani, and S. Weiss, "Vision-Controlled Micro Flying Robots : from System Design to Autonomous Navigation and Mapping in GPS-denied Environments," IEEE Robotics \& Automation Magazine, pp. 1-10, 2013.

[7] A. Briod, A. Klaptocz, J.-C. Zufferey, and D. Floreano, "The AirBurr: A flying robot that can exploit collisions," in 2012 ICME International Conference on Complex Medical Engineering (CME). Ieee, Jul. 2012, pp. 569-574.

[8] A. Briod, "Observation of Insect Collisions," Harvard University, Tech. Rep., 2008.

[9] A. Klaptocz, "Design of Flying Robots for Collision Absorption and Self-Recovery," Ph.D. dissertation, Ecole Polytechnique Fédérale de Lausanne, 2012.

[10] A. Klaptocz, A. Briod, L. Daler, J.-c. Zufferey, and D. Floreano, "Euler Spring Collision Protection for Flying Robots," in IEEE International Conference on Robotics and Automation, 2013, p. 7.

[11] A. Klaptocz, L. Daler, A. Briod, J.-C. Zufferey, and D. Floreano, "An Active Uprighting Mechanism for Flying Robots," IEEE Transactions on Robotics, vol. 28, no. 5, pp. 1152-1157, Oct. 2012.

[12] N. Rao, S. Kareti, W. Shi, and S. Iyengar, Robot navigation in unknown terrains: Introductory survey of non-heuristic algorithms, 1993.

[13] R. Siegwart, K. O. Arras, S. Bouabdallah, and N. Tomatis, "Robox at Expo.02: A large-scale installation of personal robots," Robotics and Autonomous Systems, vol. 42, no. 3-4, pp. 203-222, Mar. 2003.

[14] T. Palleja, M. Tresanchez, M. Teixido, and J. Palacin, "Modeling floor-cleaning coverage performances of some domestic mobile robots in a reduced scenario," Robotics and Autonomous Systems, vol. 58, no. 1, pp. 37-45, Jan. 2010.

[15] M. Rude, "A flexible, shock-absorbing bumper system with touchsensing capability for autonomous vehicles," Proceedings of IEEE/RSJ International Conference on Intelligent Robots and Systems. IROS '96, vol. 2, pp. 410-417, 1996.

[16] H. Yanco, "Wheelesley: A robotic wheelchair system: Indoor navigation and user interface," Assistive technology and artificial intelligence, pp. 256-268, 1998.

[17] P. S. Girão, P. M. P. Ramos, O. Postolache, and J. Miguel Dias Pereira, "Tactile sensors for robotic applications," Measurement, vol. 46, no. 3, pp. 1257-1271, Apr. 2013.

[18] M. Fend, H. Yokoi, and R. Pfeifer, "Optimal Morphology of a Biologically-Inspired Whisker Array on an Obstacle-Avoiding Robot," Advances in Artificial Life, pp. 771-780, 2003.

[19] F. Mondada, M. Bonani, X. Raemy, J. Pugh, C. Cianci, A. Klaptocz, S. Magnenat, J.-C. Zufferey, D. Floreano, and A. Martinoli, "The e-puck, a robot designed for education in engineering," in 9th Conference on Autonomous Robot Systems and Competitions, Castelo Branco, Portugal, 2009. 